\title{
OUTCOMES OF STROKE PATIENTS DISCHARGED FROM AN IN-PATIENT FACILITY IN THE EASTERN CAPE, SOUTH AFRICA: A MIXED METHODS DESIGN
}

\begin{abstract}
Statistics have indicated that stroke is a major health concern in the Eastern Cape Province in South Africa. The aim of this study was to determine the outcome of stroke patients in this setting. A concurrent mixed methods design was used. A convenient sampling technique was used to recruit participants. The Barthel Index was used to determine activity limitations, while the Modified Rankin Scale was used to measure participation restrictions. In addition the Facilitators and Barriers Survey was used to determine the environmental barriers to, and facilitators of, participation experienced by the participants. For quantitative data means, standard deviations, frequencies and percentages were calculated for descriptive purposes. Qualitative analysis was done using thematic analysis.

The mean Barthel Index score was 81,5 and the family care domain of the Modified Rankin Scale was the most affected. Participants experienced participation restrictions and activity limitations due to stairs, gravel surfaces and kerbs. The use of assistive devices and physiotherapy interventions were highlighted as facilitators to participation. The findings from the qualitative interviews supported these findings.

The study highlights the positive role of physiotherapy in the rehabilitation of stroke patients. The interventions should, however, include addressing the physical environmental barriers experienced by the patients.
\end{abstract}

\section{INTRODUCTION}

Stroke is the leading cause of serious long term disability (American Heart Association 2008). Disability poststroke is conceptualised as activity limitations and participation restrictions experienced by individuals (WHO 2001). Considering the interaction of the individual with the environment is also important when conceptualising disability. The most common activity limitations experienced by stroke survivors include difficulties with mobility

\section{Corresponding author}

Prof Anthea Rhoda

Department of Physiotherapy

University of the Western Cape

Private Bag X17

Bellville

Tel: +27 219592542

Email:arhoda@uwc.ac.za and conducting activities of daily living (Rhoda 2012; Rouillard et al 2012). Common participation restrictions experienced by these same individuals are a change in the ability to return to work (Barclay et al 2012, Mayo et al 2012), as well as leisure activities and education (Desrosiers et al 2006).

The physical environment poses the most common barrier to participation for people with physical disabilities, including those who have experienced a stroke (O'Donovan et al 2009). Hamel et al, (2006) have identified the individual barriers that arise post-stroke from personal issues in four main areas, namely: physical, social, cognitive and psychological. Physical and cognitive impairments have been frequently identified as the most important reasons for lack of participation (Hamel et al 2006). The identification of the barriers and facilitators post-stroke is crucial if the activ- ity levels and community reintegration of stroke patients are to be improved. A reduction in activity levels and participation in patients with stroke limits their reintegration into the community and they are therefore socially isolated, which often results in the patients becoming frustrated and even depressed (Dowswell et al 2000).

Correct management during the acute period post-stroke will result in less disability and reduce the negative impact on the healthcare sector and society (McNaughton et al 2005). Rehabilitation is the process through which the disability experienced by stroke patients is addressed. The rehabilitation of stroke patients could include early physio-, occupational and speech therapy, facilitating reintegration of patients with stroke into their communities, education of patients and their families about stroke and prevention of further com- 
plications. In developed countries rehabilitation can be conducted in different settings, which include being in-patients in hospitals, in rehabilitation units in acute care hospitals, in nursing facilities that offer structured rehabilitation programmes, in out-patient facilities, or in patients' homes (Greshan et al 2004). Rehabilitation improves the quality of life of stroke survivors (Kalra et al 2004; Patel et al 2004). While rehabilitation improves both the physical and the social function of patients post-stroke, it is recommended that it continues post-hospital discharge in order to maintain the level of improvement that patients have reached (Aprille et al 2008; Hopman and Verner 2003).

Although data is available on the outcomes and profile of patients with stroke in the Western Cape, South Africa (Rhoda et al 2006; Joseph and Rhoda 2013, Roullard et al 2012), no data is available for the Eastern Cape. It has been highlighted by Connor and Bryer (2005) that, due to socio-economic differences and past political influences, the profile of stroke could differ between the various population groups. Eastern Cape Mortality Estimates (MRC, 2000) indicated stroke as the leading cause of death in the Eastern Cape at 6,5\%. According to those statistics, stroke was the leading cause of death among women and the fourth leading cause among men in the age group 45-49 years. In the age group 60 years and older, stroke was the leading cause of death in both sexes, accounting for $19 \%$ of female and $11,9 \%$ of male deaths in this age group (MRC, 2000). This confirms that stroke is a serious medical condition in this region and therefore needs to be managed properly. The aim of this study was therefore to determine and explore the outcomes of stroke patients admitted to the Uitenhage Provincial Hospital from 1 January 2008 to 31 December 2009. Outcomes investigated in this study included activity limitations, participation restrictions and the environmental barriers to, and facilitators of, participation experienced by the participants.

\section{METHODS}

\section{Research Design}

A concurrent mixed model design was used to collect data. The quantitative part of the study had a retrospective and a prospective component. The retrospective part of the study collected information from medical records of admitted stroke patients that were referred for physiotherapy while hospitalised, while the prospective part of the study collected data relating to the activity limitations and participation restrictions postdischarge. Face-to-face, semi-structured interviews were conducted to collect qualitative data. Only information gathered from the quantitative prospective study and the qualitative interviews will be presented in this paper.

\section{Research Sample}

A convenient sample of stroke patients who were admitted to hospital between 1 January 2008 and 31 December 2009 were recruited, and their folders were perused to collect quantitative data. Participants were excluded from the study if they; had strokes like symptoms resulting from tumors, or other neurological conditions, such as head injuries or had severe cognitive and/or speech impairments.

\section{Research Instruments}

The Barthel Index was used to assess activity limitations (Mahoney \& Barthel, 1965). Participation restrictions were measured using the structured interview of the Modified Rankin Scale (Wilson et al., 2005). This instrument includes specific questions relating to participation in work activities, family life and leisure activities. The Facilitators and Barriers

Table 1: Participation Restrictions

\begin{tabular}{|l|l|l|}
\hline Work Ability $(\mathbf{n}=\mathbf{8})$ & No & $\%$ \\
\hline Reduced level of work & 4 & $16.7 \%$ \\
\hline Unable to work & 4 & $16.7 \%$ \\
\hline Family Care $(\mathbf{n}=\mathbf{1 5})$ & & \\
\hline Reduced responsibility in looking after family & 13 & $54.2 \%$ \\
\hline Unable to look after family & 2 & $8.3 \%$ \\
\hline Social Activities (n = 11) & & \\
\hline Participation much less/less than half as often & 6 & $25.0 \%$ \\
\hline Unable to participate & 5 & $20.8 \%$ \\
\hline
\end{tabular}

Survey (FABS) of environmental influences on participation among people with lower limb mobility impairments and limitations (Gray et al 2008), was used to determine the environmental barriers to, and facilitators of, participation experienced by the participants. The FABS includes 65 questions, 177 items and six domains that could influence participation. These domains include use of mobility assistive devices; the home environment; features of the community; ability to access destination; facilities available in the community; and community support network. The abovementioned instruments were all valid and reliable (Finch, 2002), and had previously been used in South African studies.

\section{Data Collection Procedures.}

Before the study commenced, permission and ethical clearance was obtained from the relevant senate committee at the University of the Western Cape, ethical clearance number $10 / 1 / 23$. In addition, permission to conduct the study was obtained from the Medical Superintendent of the Uitenhage Provincial Hospital. The records of stroke patients who were hospitalised from 1 January 2008 to 31 December 2009 were accessed via the Central Records Department of the Hospital. Out of a total of 168 patients whose folders were perused, only 24 patients were successfully contacted and agreed to take part in the prospective study. On the day of the interview which was previously arranged, the researcher discussed the aim of the 
study and obtained written informed consent from the patient or their primary caregivers. Participation in the study was voluntary and any participant was allowed to withdraw from the study at any stage. The participants were ensured that confidentiality would be maintained and that the researcher would be the only person who would have access to the digital recordings and that their names would not appear in any documentation published. The Barthel Index, the Modified Rankin Scale and the FABS, were then completed. All instruments used were translated from English into both Afrikaans and isiXhosa and back translated into English by two independent people. The majority (20) of the interviews were conducted at the patients' homes, while four were conducted at a nearby Community Health Centre. The interviews conducted at the Community Health Centre were completed in a private room. The questionnaires took approximately one hour to complete.

The qualitative interviews were conducted after the quantitative interviews by the researcher. The aim of the interviews was to explore the barriers and facilitators to participation of the participants. The participants who were part of the quantitative prospective study were contacted telephonically to ascertain their willingness to be interviewed. The qualitative interviews were conducted at times and places convenient to the participants. Many participants preferred to have the interview conducted

Table: 2: Barriers (Home/Community) and Effect of Barrier on Participation ( $n=24)$

\begin{tabular}{|l|c|c|c|}
\hline Barrier & $\begin{array}{l}\text { Limits } \\
\text { a lot }\end{array}$ & $\begin{array}{l}\text { Limits } \\
\text { some }\end{array}$ & $\begin{array}{l}\text { Total/ } \\
\text { Percentage }\end{array}$ \\
\hline Stairs home & 6 & 6 & $12(50 \%)$ \\
\hline Gravel home & 6 & 5 & $11(46 \%)$ \\
\hline Outside kerb (home) & 4 & 11 & $15(66 \%)$ \\
\hline Community kerb & 4 & 11 & $15(66 \%)$ \\
\hline Gravel community & 5 & 6 & $11(46 \%)$ \\
\hline Summer weather & 3 & 9 & $12(50 \%)$ \\
\hline Rainy weather & 8 & 10 & $18(75 \%)$ \\
\hline Noise levels & 4 & 5 & $9(37 \%)$ \\
\hline Crowds & 4 & 4 & $8(33 \%)$ \\
\hline
\end{tabular}

at their homes. One patient preferred to have the interview conducted telephonically. Telephonic consent was obtained to conduct the interview and to have it recorded. The qualitative interviews were recorded by means of a digital voice recorder, but where the patients did not give their consent for the recording, detailed field notes were made during and immediately after the interview. Four of the participants gave consent to be recorded, while the remaining ones refused digital recording. An interview guide was used to guide the interviews. The interview guide was developed based on literature (Wood et al 2010) and was aimed at further exploring the

Table 3: Participants' Perception of the effect of service on Participation

\begin{tabular}{|l|c|c|c|c|c|c|}
\hline $\begin{array}{l}\text { Person } \\
\text { Rendering } \\
\text { Service/ } \\
\text { Assistance }\end{array}$ & \multicolumn{6}{|c|}{ Perceptions of effect of service on participation } \\
\hline & HAL & HS & NOEF & LS & LAT & TOTAL \\
\hline Doctor/Clinic Sister & 18 & 4 & & 1 & 1 & 24 \\
\hline Therapists & 8 & 2 & 1 & & & 11 \\
\hline $\begin{array}{l}\text { Paid Personal } \\
\text { Assistant }\end{array}$ & 1 & 0 & 0 & 0 & 0 & 1 \\
\hline Family Members & 21 & 1 & 0 & 1 & 0 & 23 \\
\hline Friends & 7 & 0 & 0 & 1 & 0 & 9 \\
\hline Peers & 2 & 0 & 0 & 1 & 0 & 3 \\
\hline Store Clerks & 2 & 1 & 0 & 0 & 0 & 3 \\
\hline
\end{tabular}

Key: HAL=Helps a lot, $\mathrm{HS}=$ Helps somewhat, NOEF=No effect, LS=Limits somewhat, LAT=Limits a lot 
into English. The voice recordings were compared with the field notes several times in order to verify accuracy. The data was coded and categorised within specific predetermined themes that related to activity limitations and participation restrictions.

\section{RESULTS}

\section{Recruitment and Socio- Demographic factors of the participants}

A total of 461 patients with stroke were admitted to the Provincial Hospital in the Eastern Cape between 1 January 2008 and 31 December 2009. Of these, 181 were excluded, based on the study exclusion criteria, while 112 patient folders were missing. Retrospective data was collected from the medical folders of 168 participants. The study sample consisted of more females $(59 \%)$ than males $(41 \%)$. The mean age of the participants was 61,5 years, the SD was 13,8 , and ages ranged from 20 to 90 years. Of the 168 participant folders obtained, only $24(14,3 \%)$ participants could be followed up and agreed to be interviewed for the prospective part of the study. More than $60 \%$ of the cohort could not be reached via the telephone number listed in the medical records. Approximately $10 \%$ of the participants had died. Nine of the remaining 24 participants were conveniently selected for the qualitative part of the study.

\section{Activity Limitations}

The majority of the participants scored between 61-100 on the Barthel Index, with a mean score of 81,5 and a standard deviation of 27,9. Although the participants scored average to high on the Barthel Scale, challenges were experienced with mobility and conducting activities of daily living, as is illustrated by the comments quoted below.

P7 "Since the stroke, I do not go out much, because I cannot walk too far; I get tired easy."

P6 "My balance is at this moment my biggest problem, it makes me scared of falling, and I have little self-confidence after the stroke."

A lack of, or inability, to groom themselves limited their social activity. A female participant made specific mention of her appearance and her lack of ability to manage her own hair care, as was expressed in the quotation below:

P2 "My appearance keeps me away from many things. My hair is not nicel right and I cannot see to it myself. Some people speak out of turn and leave comments about my hair or appearance. I therefore would rather stay at home."

\section{Participation Restrictions}

The participants experienced participation restrictions in the domains of work, the ability to care for their families and to participate in social activities (see table 1). The information only relates to those participants who had the roles prior to their stroke.

The domain mostly affected by the participants was related to caring for family. A total of $15 / 24(62.5 \%)$, indicated that the ability to conduct this activity was affected post-stroke.

The change in engagement in social activities was expressed during the qualitative interviews. Social isolation affected the participants psychologically, as is illustrated in the quotation below

P3 "Family and friends do not come and visit me anymore. I was last out of this house two years ago... I only sit at home... I go nowhere. It makes me sad and depressed."

\section{Facilitators and Barriers experienced by the participants Use of mobility assistive devices.}

Over $40 \%$ of the cohort did not use any mobility devices when going out of the home or into the community. A total of $21 \%$ of the participants made use of canes/quadripods, while $17 \%$ used manual wheelchairs. Of the participants who used mobility devices, $79 \%$ stated that they always used these when they had to move about in their homes or the community, and $86 \%$ said that the devices helped them considerably to be mobile in their environment.

\section{Physical Environmental Barriers}

Stairs, kerbs and gravel surfaces were environmental features mentioned by the participants that acted as a barrier to their participation. Seventy five percent (18) of the participants indicated that rainy weather limited their participation.

Uneven surfaces were mentioned in the qualitative interviews as a factor which affected mobility and resulted in a fear of falling, which resulted in a dependency on others

P1 "I can walk outside, but the gravel scares me; I walked one day and my foot slipped over the gravel - I landed on my bum."

A commonly mentioned occurrence was the participants' ability to manipulate physical environmental features only with the help of another person. They mentioned their physical as well as psychological inability to interact with these environmental features independently, as illustrated by the following quotation:

P6 "I can climb the steps, but I need to have someone behind me; I am scared to climb the steps by myself; I am scared that I will get hurt."

P2 "Since the stroke, climbing stairs is difficult: I need to have someone to help me up the stairs. If there is no-one at home, then I do not go up the stairs."

\section{Environmental Facilitators Services and attitudes}

The following section will present the participants' perceptions of the effect of services received on participation and the effect that the attitudes of the persons rendering the service have had on the participant or participants.

Services received from the doctor and family members were seen as facilitators by most of the participants in this study. Only eleven of the participants interviewed had continued with physiotherapy post-discharge from hospital. Nine of those found physiotherapy to be a facilitator in their participation.

Many participants commonly stated that physiotherapy post-stroke had helped them with balance retraining and to walk again, as illustrated by the following quotations:

P4 "The physiotherapist taught me to walk again using a stick, and also with dressing and undressing."

P9 "The therapy helped me to walk, I can go to church again, now that I can walk alone".

Support provided by family members played a great role in participation of patients' post- stroke. Information obtained via the qualitative interviews highlighted that social support by fami- 
lies played a role in participation of stroke patients. A lack of social support affected the participants psychologically, as is illustrated in the excerpts below.

P3 "Family and friends do not come and visit me any more. I was last out of this house two years ago... I only sit at home... I go nowhere. It makes me sad and depressed."

Most of the participants in this study found that the attitudes of the different service providers had facilitated participation. Attitudes of family members were highlighted by the majority of the participants as "helping a lot". A participant highlighted a change in attitudes from family and friends, as is expressed in the following quotation

P8 "People/family do not want to help us by driving us around... When they see it's me calling, they don't answer the phone."

\section{DISCUSSION}

\section{Activity Limitations}

A mean score on the Barthel Index of 81,5 and a standard deviation of 27,9 , indicated that the majority of the participants needed minimal to moderate assistance with conducting basic activities of daily living as measured quantitatively (Granger et al 1979). Qualitative responses, however, revealed that challenges with walking and grooming resulted in participants being socially isolated. A lack of mobility and loss of upper limb function had previously been found to be factors contributing to re-engagement in active activities (O'Sullivan \& Chard, 2010) and resulted in increased difficulty in community participation (Yang \& Sanford, 2012).

\section{Participation Restrictions}

The majority of the interviewed participants mentioned a change in their ability to care for their family post- stroke. An inability to fulfil previous roles leads to dissatisfaction and individuals become upset when they see others assume the roles and responsibilities that they can no longer perform (Wood et al 2010). Social isolation, as a result of a lack of social support and the decreased ability to engage in social activities, was evident in the findings of the current study. The role change, as well as the decreased social interaction, is a common finding in stroke survivors (Barclay et al 2012). Social isolation as well as role change can lead to a myriad of feelings consisting of being a burden, helpless, frustrated, depressed, sad and angry (Dowswell et al 2000).

\section{Environmental Factors \\ Environmental Facilitators}

Over $86 \%$ of participants mentioned that the mobility device that they used served as a facilitator. Similar results were found in a study by Alguren et al, (2009), in which participants perceived walking devices, wheelchairs or other assistive devices as facilitators. Although not investigated in the current study, the provision of accessibility to assistive devices by people with disabilities in developing countries, could affect their re-integration into their homes and communities.

Interventions by both health professionals and family members were found to be a facilitator to participation by the study participants. A study by Olsson and Sunnerhagen (2006) supported the aforementioned statement, in that participants in that study recorded improvements post-stroke as a result of the rehabilitation that they had received. The participants in the current study mentioned that physiotherapy had helped to make them more functionally active within their home and community environment, similar to the findings in studies by Dowswell et al, (2009). The positive role of physiotherapy in the rehabilitation of stroke patients is once again highlighted in this study.

\section{Environmental Barriers}

The home and community physical environmental barriers that were frequently reported by the study participants, were stairs, kerbs and gravel surfaces. Similar findings were reported in studies internationally and in other African countries, which established that features of land forms, such as hills, were common barriers for more than half of the study population (Alguren et al., 2009; Yang \& Sanford, 2012; Urimmubenshi \& Rhoda 2011). The physical environment was found in some studies to be the most common barrier to participation (O'Donovan et al., 2009; Levasseur et al 2008). For many individuals, the outside of the home poses barriers, with key issues including uneven ground, poor lighting, and stair access (Reid, 2004). Therefore, environmental features, such as steps and kerbs, are significantly correlated to dependence, leading to decreased activity (Yang \& Sanford, 2012). This reduction in activity levels and participation could, conversely, result in the participant becoming increasingly isolated socially (Dowswell et al., 2000).

\section{CONCLUSION}

This mixed methods study identified that stroke patients in the Eastern Cape had activity limitations related to mobility, and participation restrictions related to caring for their families. They also experienced social isolation and identified physical environmental barriers as a major factor limiting participation. The use of assistive devices and physiotherapy interventions were seen as facilitators to participation and reintegration post-stroke. 


\section{REFERENCES}

Alguren B, Lundgren-Nilsson A, Sunnerhagen, KS. 2009 Facilitators and barriers of stroke survivors in the early post-stroke phase. Disability and Rehabilitation 31(19):1584-1591.

American Heart Association 2008 Heart Disease and Stroke Statistics. Our Guide to current statistics $\&$ supplement to our Heart and Stroke facts.

Aprille I, Di Stasio E, Romitelli F,Lancellotti S, Caliandro P, Gilardi A, Padua L 2008 Effects of rehabilitation on quality of life in patients with chronic stroke. Brain Injury 22(6):451-456.

Barclay-Goddard R, Ripat J, Mayo N 2012 Developing a model of participation post-stroke: a mixed-methods approach. Quality of Life Research 21: 417-426.

Connor MD, Bryer A 2005 Stroke in South Africa. Ed: Steyn K and Fourie J. Chronic Diseases of Lifestyle in South Africa since 1995-2005 Technical Report. Medical Research Council of South Africa, Tygerberg. Chapter 14:195-203.

Desrosiers J, Rochette A, Norea L, Boubonnaise D, Bravo G, Bourget A, 2006 Long-term changes in participation after stroke. Topics in Stroke Rehabilitation 13(4):89-97.

Dowswell G, Dowswell T, Lawler J, Green J, Young J 2002 Research Letter: Patients and caregivers' expectations and experiences of a physiotherapy intervention 1 year following a stroke: a qualitative study. Journal of Evaluation in Clinical Practice 8(3):361-365.

Dowswell G, Lawler J, Dowswell T, Young J, Forster A, Hearn J, 2000 Investigating recovery from stroke: a qualitative study. Journal of Clinical Nursing 9(4):507-515.

Finch E, Brooks D, Stratford PW, Mayo NE 2002 Physical rehabilitation outcome measures: A guide to enhanced clinical decision making (2nd Edition). Hamilton: BC Decker.

Gray DB, Hollingsworth HH, Stark S, Morgan K 2008 A Subjective measure of environmental facilitator and barriers to participation for people with mobility limitations. Disability and Rehabilitation 30:(6) 434-457.

Granger CV, Devis LS, Peters MC, Sherwood CC, Barret JE 1979 Stroke rehabilitation: analysis of repeated Barthel Index measures. Archives of Physical Medicine Rehabilitation 60:14-17.

Greshan GE, Duncan PW, Stason WB 2004 Post stroke rehabilitation. Diane Publishing.

Hamel J, Jones R, Gossett A, Morgan E 2006 Examining Barriers and Supports to Community Living and Participation After a stroke from a Participatory Action Research Approach. Topics in Stroke Rehabilitation 13(3):43-58.

Hopman WM, Verner J 2003 Quality of life during and after in-patient stroke rehabilitation. Stroke 34: $801-805$.
Kalra L, Evans A, Perez I, Melbourn A, Patel A, Knapp M, Donaldson M 2004 Training carers of stroke patients: randomised controlled trial. British Medical Journal. 328;1099.

Joseph C, Rhoda A 2013 Activity limitations and factors influencing functional outcome of patients with stroke following rehabilitation at a specialised facility in the Western Cape. African Health Sciences 13(3): 646-654.

Levasseur M, Desrosiers J, Tribble D 2008 Subjective QOL predictors of older adults with physical disabilities. American Journal of Physical Medicine \& Rehabilitation 87:830-841.

Mahoney FI, Barthel DW 1965 Functional evaluation: the Barthel Index. Maryland State Med Journal; 14:56-61.

Mayo N, Bronstein D, Scott SC, Finch LE, Miller S 2013 Necessary and sufficient causes of participation and philosophical perspectives. Quality of Life Research DOI 10.1007/s11136-013-0441-6.

McNaughton H, De Jong G, Smout RJ, Melvin J, Brandstater M 2005 Comparison of stroke rehabilitation practice and outcomes between New Zealand and United States facilities. Archives of Physical Medicine Rehabilitation 86, Sup. 2:S101-S114.

MRC 2000 South African National Burden of Disease Study Estimates of Provincial Mortality Eastern Cape Province.

O’Donovan M, Doyle A, Gallagher P 2009 Barriers, activities and participation: Incorporating ICF into service planning datasets. Disability and Rehabilitation 31(25):2073-2080.

Olsson BG, Sunnerhagen KS 2006 Effects of Day Hospital Rehabilitation After Stroke. Journal of Stroke and Cerebrovascular Disease Vol. 15, No. 3, 106-113.

O'Sullivan C, Chard G 2010 An Exploration of participation in leisure activities post stroke. Australian Occupational Therapy Journal. 57: 159166.

Patel A, Knapp M, Evans A, Perez I, Kalra L 2004 Training care givers of stroke patients: economic evaluation. British Medical Journal 328:1102.

Rhoda A 2012 Limitations in Activity and Participation experienced by stroke patients: A Qualitative Inquiry. South African Journal of Physiotherapy 68(1):1-5.

Rouillard S, Willy De Weerdt W, De Wit L, Jelsma J 2012 Functioning 6 months post-stroke after discharge from an in-patient rehabilitation facility. South African Medical Journal 6(102).

Reid D 2004 Accessibility and usability of the physical housing environment of seniors with stroke. International Journal of Rehabilitation Research 27(3): 203-208.
Urimubenshi G, Rhoda A 2011 Environmental barriers experienced by stroke patients in Musanze district in Rwanda: a descriptive qualitative study. African Health Sciences 11:3 398-408.

Wilson JT, Hareendran A, Hendry A, Potter J, Bone I, Muir KW 2005 Reliability of the Modified Rankin Scale Across Multiple Raters: Benefits of a Structured Interview. Stroke 36:777-781.

Wood JP, Connelly DM, Maly MR, 2010 Getting back to real living: a qualitative study of the process of community reintegration after stroke. Clinical Rehabilitation 24:1045-1056.

World Health Organization 2001 International Classification of Functioning Disability and Health (ICF). Geneva, Switzerland: WHO.

Yang H, Sanford JA 2012 Home and Community Environmental Features, Activity Performance and Community Participation among Older Adults with Functional Limitations. Journal of Ageing Research, doi:10.1155/2012/65758. 